\title{
Programa de formación cultural en la CREB de Venero ${ }^{1}$
}

\section{Cultural training program in the CREB of Venero}

DOI: http://dx.doi.org/10.17981/cultedusoc.9.3.2018.79

Artículo de investigación. Fecha de recepción: 15/06/2018. Fecha de aceptación: 27/11/2018

\author{
Maira Garcés²; \\ Álvaro Robles; Anis Escobar; Edgardo Dunoyer; Elvis Payan; \\ Evangelista Rocha; Ereidis Guillén; Henry Echeverría; Juan Olivares; \\ Liliana Rojas; Maira Garcés; María Mejía; Orlando Pérez; \\ Sandra Garcés; Sandra Escobar y Yasmin Rocha ${ }^{3}$ \\ IED Andrés Díaz Venero De Leiva (Colombia) \\ maigara3477@gmail.com
}

Para citar este artículo:

Garcés, M., Robles, A., Escobar, A., Dunoyer, E., Payan, E., Rocha, E., Guillén, E., Echeverría, H., Olivares, J., Rojas, L., Garcés, M., Mejía, M., Pérez, O., Garcés, S., Escobar, S. y Rocha, Y. (2018). Programa de formación cultural en la CREB de Venero. Cultura. Educación y Sociedad 9(3), 667-676. DOI: http://dx.doi.org/10.17981/cultedusoc.9.3.2018.79

\section{Resumen}

Las actividades culturales se han convertido en instrumento que potencia y estimula capacidades y habilidades en los estudiantes. Es por ello que el presente estudio tiene como objetivo principal Elaborar un programa de formación cultural de la ERM de Venero. Haciendo énfasis a la estructuración, desarrollo y cambio de los proyectos culturales en Latinoamérica y en específico en Colombia. La investigación es de tipo Cualitativa, bajo un alcance descriptivo empleando como instrumento la entrevista estructurada, y fue aplicada miembros de la comunidad educativa IED Externado de San Sebastián en el Municipio de San Sebastián Magdalena, Colombia. Como conclusión se puede afirmar que Los estudiantes se motivan a participar en todos los eventos culturales, ya sea en grupos de danzas ya establecidos o creando algunos dentro de la comunidad para poder así mostrar todos los tipos de bailes típicos de la región.

Palabras clave: Programa, Formación Cultura; actividades culturales; proyectos culturales.

\section{Abstract}

Cultural activities have become an instrument that encourages and stimulates skills and abilities in students. For this reason, the main objective of this study is to prepare a cultural training program for Venero's ERM. Emphasizing the structuring, development and change of cultural projects in Latin America and specifically in Colombia. The research is Qualitative, under a descriptive scope using as an instrument the structured interview, and was applied to members of the educational community of the Externado IED of San Sebastián in the Municipality of San Sebastián Magdalena, Colombia. In conclusion it can be affirmed that students are motivated to participate in all cultural events, either in groups of established dances or creating some within the community to be able to show all the typical dances of the region.

Keywords: Program, Culture Training; cultural activities; cultural projects.

\footnotetext{
1 Este artículo ha sido derivado del Programa de Fortalecimiento de la Cultura Ciudadana y Democrática CT+I a través de la IEP apoyada en TIC en el Departamento de Magdalena: CICLON.

2 Líder del grupo de investigación "Danzando ando".

3 Docentes pertenecientes al grupo de investigación "Danzando ando" de la IED Andrés Díaz Venero De Leiva. Proyecto Ciclón. Estrategia $\mathrm{N}^{\circ} 2$. Universidad de la Costa CUC.
}

- The author; licensee Universidad de la Costa - CUC. 


\section{Introducción}

El mundo Globalizado y la dinámica acelerada de la información, así como el vertiginoso avance de las tecnologías de la información y comunicación han llevado a que el acceso al conocimiento de otras culturas y tradiciones sea de fácil alcance. Sin embargo, resulta existen una variedad de sistemas sociales, que no son necesariamente homogéneos, sino más bien heterogéneos, y que tienen características marcadamente modernas, lo cual se evidencia en las distintas culturas. (Herrera; Certain y Calderón. 2014). De acuerdo a lo anterior es común ver como los estudiantes con el pasar del tiempo se han adaptado a los cambios y se han apropiado de modas y patrones culturales foráneos, aislando de ellos las raíces Culturales y folclóricas autóctonas, y dejando en el olvido los valores e identidad cultural.

Abarcar procesos de diversidad cultural, permite que los jóvenes aprendan a darle valor a su cultura, con el fin de preservarla e impulsar su desarrollo lo cual permite también que sean los maestros los que se involucren, "siendo ellos conocedores de las representaciones iniciales de los alumnos, de sus saberes previos y de su contexto, a fin de estimularlos a conocer, comprender y valorar su propia comunidad, reforzar su autoestima e identidad personal y cultural" (Durán y Parra, 2013).

El CREB de Venero busca impulsar, y fortalecer los procesos y actividades culturales de interés común en los estudiantes a través de la elaboración de un programa dirigido a los niños, niñas y jóvenes de la institución educativa que lidere el proceso de visibilizar la identidad cultural de la comunidad.

Por esto se propone implementar el desarrollo del Programa de formación cultural del CREB de Venero, la formación de docentes y estudiantes en danzas y cantos tradicionales; con el objetivo de que estas costumbres perduren en el tiempo a través de generaciones. Con el apoyo del grupo de investigación cultural "Danzando Ando", inicialmente con el encuentro estudiantil de danzas; para luego llegar a la institucionalización del festival De Cantos y Danzas Tradicionales a nivel regional, nacional e internacional. Es importante reconocer que la identidad cultural genera sentido de pertenencia y contribuye al fortalecimiento de valores autóctonos.

En 1982, la UNESCO llevo a cabo en México la "Conferencia Mundial sobre las Políticas Culturales", donde la comunidad internacional de expertos suscribió la siguiente declaración, que podría ser utilizada como referencia para elaborar cualquier proyecto cultural.

La cultura puede ser considerada como "el conjunto de los rasgos distintivos, espirituales y materiales, intelectuales y afectivos que caracterizan una sociedad o un grupo social." También hace parte de los modos de vida, los derechos fundamentales del ser humano, los sistemas de valores, las tradiciones, las creencias y le brinda al individuo el poder reflexionar sobre el mismo. La cultura es la que hace a los seres humanos, racionales, críticos y éticamente comprometidos. A través de ella discernimos los valores y efectuamos opciones. A través de ella el individuo puede expresarse, toma conciencia de sí mismo, pone en cuestión sus propias realizaciones, busca continuamente nuevas significaciones, $\mathrm{y}$ crea obras que lo trascienden.

$\mathrm{Al}$ respecto, Romero y Contreras (2015) destacan la necesidad de que los individuos al coexistir como sujetos dentro de la sociedad desarrollen capacidades para la toma de decisiones en función de su proyecto de vida.

A continuación se hace mención de algunos proyectos que se desarrollan a nivel internacional, nacional, regional y municipal.

A nivel internacional la Pontificia Universidad católica del Ecuador sede Ibarra implemento el proyecto la danza en el ecua- 
dor y el mundo, desarrollado por Ana Córdoba propone que la danza es la identidad histórica, autónoma de la humanidad basándose en el principio de que la mayoría de las sociedades viven la vida como una danza.

A nivel Nacional existe una gran variedad de festival que manifiesta la diversidad cultural de Colombia. Entre ellos: el Carnaval de Barranquilla, Carnaval de Blancos y Negros en Pasto, festival nacional del Bambuco en Huila, festival nacional del Joropo en Villavicencio. Entre muchos más.

A nivel Regional se desarrolla en el Banco Magdalena el festival de la Cumbia, el festival de la Tambora en Tamalameque, Cesar, el festival de Dazas de Talaigua nuevo Sur de Bolívar.

A nivel Municipal se realizan el festival de Chande en San Sebastián de Buenavista Magdalena, el festival del Recuerdo en el corregimiento de Troncoso.

La problemática que se presenta en la comunidad estudiantil de la Institución Educativa Técnica Departamental Andrés Díaz Venero de Leiva, se atribuye a una serie de factores y tendencias influenciadas por modas y estilos actuales que han conllevado a debilitar la identidad cultural local y regional, lo que se visibiliza en los siguientes aspectos y/o comportamientos:

- El poco conocimiento y la falta de apropiación de las tradiciones culturales a nivel local y regional, de un alto porcentaje de los miembros de la comunidad educativa.

- Los jóvenes, son influenciados por modas y patrones culturales externos dejando de lado las raíces culturales y tradiciones propias, perdiendo con ello identidad cultural.

- El currículo se ha enfocado en el desarrollo de los contenidos de las áreas fundamentales dejando de lado el estudio y/o fortalecimiento del contexto cultural, descuidando cultivar el sentido de pertenencia.
- Influencia del medio familiar y social en el educando con ciertas actitudes de apatía por actividades que resalten la identidad cultural.

- Los medios masivos de comunicación promueven el comportamiento de aculturación del niño debido a su capacidad de recepción e imitación.

Teniendo en cuenta los anteriores aspectos, se hace necesario aplicar el proyecto del Programa de formación cultural del CREB de Venero, el cual pretende buscar un interés colectivo en los niños, niñas y jóvenes y de esta manera motivarlos a los procesos formativos de tradiciones culturales propias de la región, con la interrelación de los diferentes miembros participantes de la comunidad para fortalecer la convivencia e identidad cultural de la región.

\section{Tradiciones Culturales}

Las tradiciones Culturales son el conjunto de todas las manifestaciones de la cultura popular de una región y por lo tanto incluye las leyendas, los cuentos, las danzas, las tradiciones, la música y gran cantidad de expresiones artísticas diversas.

Cultura proviene del latín cultus, el cual hace referencia al cultivo del espíritu humano y de las facultades del hombre. También se puede definir como conjuntos de conocimientos e ideas, tradiciones y costumbres que caracterizan a un pueblo, a una clase social, en determinada época. (Min-Cultura, 1997)

El interés del pueblo es expresar supervivencias de hechos antiguos, sin importar la clase social o estamento. Se trata de la erudición del pueblo, en las supervivencias de hechos que se aprenden y han penetrado profundamente en la mentalidad popular.

El Ministerio de Cultura apoya este tipo de iniciativas para fortalecer costumbres y tradiciones de las comunidades como una estrategia para rescatar la identificación cultural que esto significa, a través de la implementación de proyectos transversales (Min-Cultura, 2013) 
Los hechos culturales se dice que son grupales porque son transmitidos en las comunidades por tradición. Se consideran populares, debido a que son el patrimonio más apreciado de los pueblos. Son propios, se exponen de manera espontánea, de forma oral. Son funcionales, se identifican con los contextos social, material y económico de la comunidad. "Son regionales, por determinada región y expresión de los modos y circunstancias locales."

\section{La Danza}

Una definición valida de la danza seria, la manera de expresar los sentimientos y emociones a través de movimientos al ritmo de la música.

"La danza es un texto cultural, una práctica social; ballet, folclor, breakdance, salsa, danza contemporánea, entre otras, son realidades de comunidades particulares en las que se producen subjetividades, se construyen identidades desde su ejercicio, pluralidades" (Olaya., Sotelo y Vargas, 2011).

Cada cultura tiene características distintivas y la danza suele ser una de ellas. Y la danza puede ser expresada de diversas maneras, pero en general, es la unión del ritmo musical con movimientos corporales.

"Se estima que la danza fue una de las primeras manifestaciones artísticas de la historia de la humanidad. La danza implica la interacción de diversos elementos. El movimiento del cuerpo requiere de un adecuado manejo del espacio y de nociones rítmicas" (Molina, 2014).

La lógica del bailarín no es más que ejecutar movimientos acompañados de la música. Por ejemplo: "una melodía de ritmo lento y tranquilo requiere de pasos de danza pausados y poco estridentes.”

Además de los movimientos, hay un factor adicional que hace parte de lo necesario durante la danza, es La expresión corporal. Es primordial saber que el predominio del ritmo o del uso del espacio dependerá del tipo de danza.
Puede haber otros factores adicionales a la danza, como la mímica y el canto, también forman parte del baile. Esta es conocida como coreografía, es una estructura de movimientos predeterminados y coordinados que se lleva a cabo a la hora de bailar. "La coreografía indica los pasos a seguir durante el baile: se trata, por lo tanto, de movimientos que no son espontáneos, sino que obedecen al diseño de un coreógrafo."

Por otro lado, Los rituales o bailes informales suelen ser espontáneos, no necesitan, ni utilizan la preparación de una coreografía. Es esos casos, cada individuo puede bailar con libertad.

La Cumbia Colombiana es el baile representativo del país, es una rica expresión dejada por los africanos. También bailes como el Zambapalo llegado de las indias orientales y que fue traída por los conquistadores al continente que son comunes en las regiones costeñas de Colombia; lo mismo que la costumbre de festejar a los santos Católicos la fiesta de la Candelaria en Cartagena, las fiestas de la Virgen del Carmen y de la Inmaculada Concepción en todo el país.

\section{Danzas Colombianas}

En Colombia las danzas surgen principalmente por medio de culturas europeas, africanas, indígenas, andinas, e igualmente, pero en menor cantidad, persas. Los bailes, y danzas de Colombia, son dependientes según la región o departamento. "La riqueza colombiana, en variedad de danzas, la convertido tradicionalmente en una nación importante a nivel cultural en Latinoamérica." (Cidoncha y Díaz 2009; Florián, 2015).

Desde un punto de vista diferente, la danza puede plantearse según el lugargenero, se presenta la inclusión y exclusión por el poder, también se presenta la definición del "deber ser". 
La política que se propone implementar por medio de las acciones en la danza que no elimina las diferencias, pero expone lo que en la actualidad argumentan los discursos que habitan y movilizan la práctica, los mismos discursos que muestran cierto grado de posibilidad para mediar la construcción de un escenario común.

"Se trata entonces de la identificación de categorías que, si bien cada género o lenguaje se apropia como nicho independiente, pueden ser tratadas como transversales o comunes, manteniendo la posibilidad de reconfigurarse y redefinirse constantemente desde el hacer mismo de la práctica" (Plan Nacional de Danza. 2010).

Se debe entender la identidad desde la multiplicidad y lo diferente que ella involucra, analizarla desde distintos enfoques, y reconocer el lugar de la mirada desde donde se nombra y qué efectos produce ese nombre, definiendo así, por ejemplo, "es posible pensar en una identidad construida desde la tradición (el folclor), pero también desde subjetividades particulares como la juvenil y, por qué no decirlo también, una identidad desde la construcción misma de los cuerpos para la danza." "Así, establecemos que una categoría que en ocasiones puede tornarse excluyente y que se utiliza para defender posiciones a ultranza, es un territorio de flujos, movilidad, inestabilidad incluso, y por lo tanto un escenario rico en posibilidades de investigación y creación” (Jiménez, 2005).

\section{Metodología}

Este estudio se llevará a cabo desde una mirada Cualitativa, de alcance descriptivo, pues tal como lo afirma (Martínez, 2006) es la investigación cualitativa la que estudia la realidad en su contexto natural, identificando la naturaleza de la realidad asociada con su comportamiento y sus manifestaciones, además, en su dimensión dinámica busca cumplir las dos tareas básicas de toda investigación: recoger la información y categorizarlos e interpretarlos.

\section{Escenarios y actores}

El proceso investigativo se llevó a cabo en la Institución Educativa Externado de San Sebastián, sede ERM de Venero. Contó con la participación de (30) miembros de la comunidad educativa. (Padres de familia, estudiantes y docentes).

\section{Técnicas e instrumentos de} recolección de la información

Se empleó como técnicas de recolección: la entrevista. De manera que la información es registrada en un instrumento de entrevista estructurada, que permiten su clasificación y proporciona facilidad para su análisis e interpretación.

\section{Procedimiento}

Momento (1): Se seleccionan los instrumentos a utilizar.

Momento (2): Se especifican las categorías de estudio.

Momento (3): Se escoge los instrumentos de recolección de información.

Momento (4): Con base a las categorías de estudio: (Cultura, comunidad, política educativa), se aplicó a los participantes la entrevista, la cual pretende describir el fenómeno que se estudia partiendo del nivel de la investigación (no experimental) en donde se observa, y analiza tal y como acontece.

Momento (5): Una vez se tiene los resultados de la entrevista estructurada y la observación registrada se interpretan y comprenden los resultados a la luz de lo que exponen los teóricos que soportan la investigación.

Momento (6): Se relacionan los resultados $\mathrm{y}$ se muestran los hallazgos.

\section{Resultados}

A continuación se muestran los resultados a la luz de las categorías de estudio abordadas. 


\begin{tabular}{|c|c|c|c|}
\hline $\begin{array}{c}\text { Categoría de } \\
\text { estudio }\end{array}$ & $\begin{array}{c}\text { Pregunta } \\
\text { orientadora }\end{array}$ & Discurso de los actores & Articulación y sistematización teórica \\
\hline Comunidad & $\begin{array}{l}\text { ¿Qué } \\
\text { manifestaciones } \\
\text { culturales } \\
\text { de nuestro } \\
\text { corregimiento } \\
\text { conoce usted? }\end{array}$ & $\begin{array}{l}\text { "25 de noviembre dia } \\
\text { de Santa Catalina, } \\
\text { Bailes populares de } \\
\text { noche buena, } \\
\text { Recocha de } \\
\text { carnavales" "Festival } \\
\text { del Chandé y del } \\
\text { recuerdo en Troncoso" } \\
\text { "Teatro, Zambapalo, } \\
\text { Cumbia" "Bullerengue" } \\
\text { "Festival vallenato" }\end{array}$ & $\begin{array}{l}\text { De acuerdo a los hallazgos encontrados en la entrevista } \\
\text { aplicada, se deduce que los estudiantes conocen muchas de } \\
\text { las manifestaciones culturales que tiene el municipio, sobre } \\
\text { todo fechas claves como el } 25 \text { de noviembre día de Santa } \\
\text { Catalina y festivales importantes como el del Chandé y del } \\
\text { recuerdo en Troncoso. } \\
\text { Frente a esto, se afirma que las tradiciones Culturales } \\
\text { es el conjunto de todas las manifestaciones de la cultura } \\
\text { popular de una región e incluye por lo tanto, las leyendas, los } \\
\text { cuentos, las danzas, las tradiciones, la música y multitud de } \\
\text { expresiones artísticas diversas. } \\
\text { Lo anterior demuestra que todas las actividades } \\
\text { mencionadas por los estudiantes son precisamente } \\
\text { manifestaciones o tradiciones culturales ya que según la } \\
\text { definición, dichas actividades concuerdan a la perfección. }\end{array}$ \\
\hline Cultura & $\begin{array}{l}\text { ¿De qué } \\
\text { manera } \\
\text { promueve } \\
\text { usted el folclor } \\
\text { nuestro en sus } \\
\text { hijos? }\end{array}$ & $\begin{array}{l}\text { "Enseñándoles los } \\
\text { diferentes bailes } \\
\text { tradicionales" } \\
\text { "Aprendan percusión } \\
\text { con los instrumentos" } \\
\text { "Aprendiendo los } \\
\text { cantos populares" } \\
\text { "Apreciar los } \\
\text { vestuarios de cada } \\
\text { danza" "Enseñándoles } \\
\text { a bailar, a tocar } \\
\text { los tambores" } \\
\text { "Aprender los cantos } \\
\text { tradicionales" } \\
\text { "Participar en } \\
\text { encuentros y } \\
\text { festivales" "Apoyando } \\
\text { y participando en los } \\
\text { eventos". }\end{array}$ & $\begin{array}{l}\text { A la luz de lo expresado por los entrevistados, se evidencia } \\
\text { que tienen claro como poder mantener el folclore a lo largo } \\
\text { del tiempo, pues solo aprendiendo bailes tradicionales, } \\
\text { tocando instrumentos tradicionales y participando de los } \\
\text { eventos tradicionales de la región es que esta cultura puede } \\
\text { mantenerse viva. } \\
\text { Las respuestas brindadas a través de la entrevista } \\
\text { contribuyen a la afirmación de que la cultura viene del } \\
\text { latín Cultus el cual hace referencia al cultivo del espíritu } \\
\text { humano y de las facultades del hombre. También se puede } \\
\text { definir como conjuntos de conocimientos e ideas, tradiciones y } \\
\text { costumbres que caracterizan a un pueblo, a una clase social, } \\
\text { en determinada época. } \\
\text { Lo anterior deja ver que existe claridad sobre la idea de } \\
\text { que la cultura cultiva el espíritu humano y esto se logra } \\
\text { precisamente avivando de todas las maneras posibles la } \\
\text { cultura de la región, ya sea con la danza, los instrumentos, } \\
\text { incluso la poesía o el canto. }\end{array}$ \\
\hline Idiosincrasia & $\begin{array}{l}\text { ¿De qué } \\
\text { manera } \\
\text { los ritmos } \\
\text { modernos han } \\
\text { incidido en la } \\
\text { decadencia } \\
\text { de los ritmos } \\
\text { tradicionales? }\end{array}$ & $\begin{array}{l}\text { "Falta de apoyo } \\
\text { del gobierno local" } \\
\text { "Incidencia de la } \\
\text { televisión e internet" } \\
\text { "Por no valorar } \\
\text { adecuadamente lo } \\
\text { nuestro" "Muchos de } \\
\text { los tamboreros han } \\
\text { fallecido o se han ido" } \\
\text { "Incidencia de ritmos } \\
\text { extranjeros en nuestra } \\
\text { cultura" "Influencia } \\
\text { de otras culturas" } \\
\text { "Por el avance } \\
\text { tecnológico" "La } \\
\text { tecnologí entretiene } \\
\text { mucho y se olvidan } \\
\text { estas costumbres" } \\
\text { "Por los medios de } \\
\text { comunicación" }\end{array}$ & $\begin{array}{l}\text { Dentro de las respuestas expuestas por los estudiantes se } \\
\text { hace notorio que la principal razón que ellos consideran por } \\
\text { lo cual se han perdido algunas tradiciones es por las TIC y } \\
\text { los Medios de comunicación, es decir la tecnología en general, } \\
\text { puesto que los jóvenes se dejan influenciar por las culturas de } \\
\text { otras partes del mundo que se propagan por estos medios. } \\
\text { Se reafirman las conclusiones explicitas por los estudiantes, } \\
\text { dado que el interés del pueblo es expresar supervivencias de } \\
\text { hechos antiguos, sin importar la clase social o estamento. } \\
\text { Se trata de la erudición del pueblo, en las supervivencias de } \\
\text { hechos que se aprenden y han penetrado profundamente en la } \\
\text { mentalidad popular. } \\
\text { Lo anteriormente dicho permite comprobar que solamente } \\
\text { mediante la tradición oral se puede enseñar a las nuevas } \\
\text { generaciones las tradiciones de la región independientemente } \\
\text { de las clases sociales, ya que todos pertenecen a la misma } \\
\text { zona y por lo tanto deben conocer sus raíces }\end{array}$ \\
\hline
\end{tabular}




\begin{tabular}{|c|c|c|}
\hline $\begin{array}{c}\text { Categoría de } \\
\text { estudio }\end{array}$ & $\begin{array}{c}\text { Pregunta } \\
\text { orientadora }\end{array}$ & Discurso de los actores \\
\hline Cultura & $\begin{array}{l}\text { ¿De qué forma } \\
\text { se fortalecen } \\
\text { los ritmos } \\
\text { modernos y } \\
\text { tradicionales? }\end{array}$ & $\begin{array}{l}\text { "Retomando } \\
\text { las costumbres } \\
\text { populares" "Creando } \\
\text { semilleros de niños, } \\
\text { jóvenes y adultos } \\
\text { que practiquen los } \\
\text { bailes tradicionales" } \\
\text { "Conseguir vestuarios } \\
\text { para participar en } \\
\text { eventos" "Con la } \\
\text { presencia de nuestros } \\
\text { compositores" } \\
\text { "Creación de nuevos } \\
\text { ritmos con mensajes } \\
\text { de la identidad } \\
\text { de nuestro folclor" } \\
\text { "Organizando talleres, } \\
\text { invitando tutores, } \\
\text { adultos mayores para } \\
\text { que enseñen a las } \\
\text { nuevas generaciones } \\
\text { a continuar con la } \\
\text { costumbre propia } \\
\text { de nuestro pueblo" } \\
\text { "Rescatando viejas } \\
\text { costumbres para que } \\
\text { no pasen de moda" }\end{array}$ \\
\hline
\end{tabular}

Los estudiantes reconocen que la mejor manera de preservar las costumbres de la región es mediante la tradición oral, apoyados en las generaciones anteriores, que sean los mismos adultos mayores quienes en espacios de enseñanza le transmitan a los jóvenes todas esas costumbres populares que se están perdiendo.

Resulta relevante anotar que los resultados de las entrevistas a los estudiantes nos expresan directamente que los hechos culturales son grupales porque una comunidad los transmite por tradición. "Son populares, debido a que son el patrimonio más querido de los pueblos. Son propios, se exponen en forma espontánea de forma oral. Son funcionales, se identifican con la vida social, material y económica de la comunidad. Son regionales, por determinada región y expresión de los modos y circunstancias locales." (Cáceres, \& Vera, 2013).

Se destaca entonces la necesidad de realizar los talleres, eventos y otras actividades invitando de tutores a los adultos mayores y los compositores que se encargan de mantener los ritmos tradicionales vivos, para que enseñen a las nuevas generaciones a continuar con la costumbre propia de nuestro pueblo y así poder rescatar las viejas costumbres para que no pasen de moda.

De acuerdo a los hallazgos encontrados en la entrevista aplicada, se deduce que los estudiantes conocen los valores culturales porque algunos de sus abuelos les cuentan las anécdotas y vivencias que tuvieron en su juventud; además de que inculcan en los más jóvenes la asistencia a las fiestas patronales como santa catalina, carnavales, festivales, etc. (Bean, 2000).

Frente a esto se debe tener en cuenta que en el siglo XVI penetró la sociedad española con un sistema de vigencias, creencias, usos, tradiciones y formas de vida de la cultura occidental cristiana. Este dominio colonial tuvo vigencia durante los siglos XVI, XVII y XVIII. Durante los años del coloniaje otro elemento penetró en la cultura social de la etnia colombiana: el negro africano, traído en esclavitud. Este hombre se localizó en las Costas Atlántica y Pacífica, Valles del Magdalena y el Cauca y regiones diversas de las minas y las haciendas.

Por lo anterior es importante recalcar que solamente mediante la tradición oral de todas estas vivencias y experiencias es que se puede mantener viva la tradición cultural de la región puesto que solamente escuchando a los adultos mayores las vivencias de ellos y de sus abuelos, y las generaciones anteriores que fueron pasando la información de generación en generación es que se pueden conocer los inicios de estas tradiciones. 


\begin{tabular}{|c|c|c|c|}
\hline $\begin{array}{c}\text { Categoría de } \\
\text { estudio }\end{array}$ & $\begin{array}{c}\text { Pregunta } \\
\text { orientadora }\end{array}$ & Discurso de los actores & Articulación y sistematización teórica \\
\hline $\begin{array}{l}\text { Política } \\
\text { educativa }\end{array}$ & $\begin{array}{l}\text { ¿Cuál crees } \\
\text { que es la } \\
\text { importancia } \\
\text { de rescatar } \\
\text { y conservar } \\
\text { la cultura } \\
\text { folclórica del } \\
\text { corregimiento? }\end{array}$ & $\begin{array}{l}\text { "Queremos que Venero } \\
\text { sea pueblo alegre, } \\
\text { lleno de música, ritmo } \\
\text { donde el hombre y } \\
\text { la mujer exponen su } \\
\text { propio estilo cultural" } \\
\text { "Es importante porque } \\
\text { la gente manifiesta } \\
\text { alegrí y entusiasmo" } \\
\text { "Porque si exponemos } \\
\text { lo nuestro, estamos } \\
\text { exponiendo nuestra } \\
\text { verdadera identidad } \\
\text { cultural" Es } \\
\text { importante porque nos } \\
\text { identifica con respecto } \\
\text { a otra tradiciones" }\end{array}$ & $\begin{array}{l}\text { A la luz de lo expresado por los entrevistados se percibe que } \\
\text { para ellos es muy importante mantener la tradición cultural } \\
\text { de la región, ya que consideran que esas son sus raíces, sus } \\
\text { principios, su identidad; y solamente conociendo eso, sienten } \\
\text { que pertenecen a algo más grande que ellos. } \\
\text { Las respuestas brindadas a través de la entrevista } \\
\text { contribuyen a confirmar que la Cultura colombiana es } \\
\text { muy rica, "tanto en el número, como en la variedad de sus } \\
\text { manifestaciones, a tal punto que cada una de las distintas } \\
\text { regiones geográficas del país, posee sus características } \\
\text { culturales propias." (Molina, 2014). } \\
\text { Es importante resaltar que si cada región de Colombia } \\
\text { tiene su propia identidad cultural entonces lo expresado por } \\
\text { los estudiantes es acertado, ya que ellos deben conocer a } \\
\text { profundidad cuales son los orígenes de las costumbres que } \\
\text { tienen, las tradiciones culturales de la región ya sea la danza, } \\
\text { la música, la poesía etc. }\end{array}$ \\
\hline $\begin{array}{l}\text { Política } \\
\text { educativa }\end{array}$ & $\begin{array}{l}\text { ¿Qué } \\
\text { estrategias } \\
\text { propone para } \\
\text { difundir } \\
\text { el folclor } \\
\text { representativo } \\
\text { de la } \\
\text { comunidad de } \\
\text { Venero? }\end{array}$ & $\begin{array}{l}\text { "Mostrar un proyecto } \\
\text { de encuentro o } \\
\text { festival de danza o } \\
\text { bailes tradicionales" } \\
\text { "Que vuelvan los } \\
\text { músicos, tamboreros } \\
\text { y bailarines a } \\
\text { mostrar su talento" } \\
\text { "Realizar el primer } \\
\text { encuentro de danza } \\
\text { Zambapalo el } 24 \text { de } \\
\text { noviembre" "Organizar } \\
\text { encuentros culturales } \\
\text { y eventos" "Crear el } \\
\text { primer encuentro de } \\
\text { baile de Zambapalo" } \\
\text { "Promocionar nuestro } \\
\text { ritmos }\end{array}$ & $\begin{array}{l}\text { Las respuestas expuestas por los estudiantes hacen notorio } \\
\text { que la principal propuesta es hacer más eventos donde los } \\
\text { músicos, tamboreros y bailarines muestren todo su talento, } \\
\text { para promocionar y socializar los ritmos culturales de } \\
\text { la región; además se necesita investigar más sobre todo } \\
\text { mediante la tradición oral, es decir, preguntar lo más posible } \\
\text { a los adultos mayores. } \\
\text { Se reafirman las conclusiones explícitas por los estudiantes } \\
\text { ya que el proyecto está basado en el enfoque introspectivo } \\
\text { vivencial, debido a que se trata de los simbolismos } \\
\text { socioculturales a través de los cuales los actores de un } \\
\text { determinado grupo social abordan la realidad (humano } \\
\text { y social fundamentalmente). Encaminado a recuperar } \\
\text { las tradiciones de las danzas y cantos tradicionales de la } \\
\text { comunidad. } \\
\text { Lo anterior permite concluir que para poder recuperar } \\
\text { todas las costumbres y tradiciones culturales de la región se } \\
\text { requiere de la participación no solo de los jóvenes deseosos } \\
\text { de aprender su identidad cultural sino también de los adultos } \\
\text { mayores y de los especialistas en esta temática como los } \\
\text { músicos, los tamboreros, los grupos de danza, etc. }\end{array}$ \\
\hline $\begin{array}{l}\text { Política } \\
\text { educativa }\end{array}$ & $\begin{array}{l}\text { ¿De qué } \\
\text { manera } \\
\text { usted como } \\
\text { miembro de } \\
\text { ésta comunidad } \\
\text { apoyaría a la } \\
\text { institución } \\
\text { educativa en } \\
\text { el proyecto } \\
\text { "Programa } \\
\text { de formación } \\
\text { cultural"? }\end{array}$ & $\begin{array}{l}\text { "Estar dispuesto } \\
\text { a participar en } \\
\text { cualquier evento } \\
\text { cultural que rescata } \\
\text { nuestras costumbres } \\
\text { perdidas" "Formar } \\
\text { grupos de danzas y } \\
\text { bailes y organizar } \\
\text { comunidades para } \\
\text { participar en eventos" } \\
\text { "Estar dispuesto a } \\
\text { participar en eventos } \\
\text { que lleven a fortalecer } \\
\text { y rescatar la danza } \\
\text { tradicional" }\end{array}$ & $\begin{array}{l}\text { Los estudiantes están dispuestos a participar en todos } \\
\text { los eventos culturales, ya sea en grupos de danzas ya } \\
\text { establecidos o creando algunos dentro de la comunidad para } \\
\text { poder así mostrar todos los tipos de bailes típicos de la región. } \\
\text { Resulta relevante anotar que la danza es la acción o manera } \\
\text { de bailar. Se trata de la ejecución de movimientos al ritmo de } \\
\text { la música que permite expresar sentimientos y emociones. Se } \\
\text { estima que la danza fue una de las primeras manifestaciones } \\
\text { artísticas de la historia de la humanidad. La danza implica } \\
\text { la interacción de diversos elementos. El movimiento del } \\
\text { cuerpo requiere de un adecuado manejo del espacio y de } \\
\text { nociones rítmicas. } \\
\text { Se destaca entonces la necesidad de que los jóvenes de las } \\
\text { nuevas generaciones hagan parte de los grupos de danzas } \\
\text { ya establecidos y reconocidos de la región o fundando nuevos } \\
\text { que puedan mostrar más ritmos diferentes de la región y así } \\
\text { poder abarcarlos todos. }\end{array}$ \\
\hline
\end{tabular}

Fuente: elaboración propia. 


\section{Discusión}

Los hallazgos encontrados con anterioridad se enmarcan en el estudio denominado: "Programa de Formación Cultural En La CREB De Venero" en donde se resaltan los aportes más relevantes:

- Los jóvenes han participado en actividades culturales en la región, pero en el marco pedagógico, expresan que es poco lo que se trabaja, por lo cual han expuesto estrategias en las cuales quisieran participar y reconocen la importancia de conocer toda la tradición cultural pues esta es su identidad.

- Los espacios en los cuales los adultos mayores y los especialistas en la tradición cultural de la región como los músicos, los tamboreros y demás se han motivado a compartir sus conocimientos con las nuevas generaciones. Estrategias como por ejemplo el que los estudiantes participen en los eventos culturales siendo ellos los que danzan, entonces puedan participar en eventos culturales de otras partes del país para dar a conocer sus tradiciones.

- Se debe diseñar estrategias pedagógicas que promueva en la comunidad educativa la articulación de la cultura y la tradición en los proyectos formativos institucionales, en donde sean los estudiantes quienes potencien habilidades y competencias desde el arte, música y danza.

\section{Referencias}

Avendaño, Cortés O, Guerrero Hilda. (2015) Competencias sociales y tecnologías de la información y la comunicación como factores asociados al desempeño en estudiantes de básica primaria con experiencia de desplazamiento forzado. Diversitas: Perspectivas en Psicología, 11(1). 13-36.
Martínez, L., Pérez, E. y Ramírez, C. (2015). La danza como estrategia pedagógica para disminuir la agresividad en los niños de preescolar de la ied el Tequendama sede Santa Rita. [Trabajo grado]. Fundación Universitaria Los Libertadores, La Mesa.

Cáceres, P. y Vera, A. (2013). Propuesta metodológica para utilizar la danza folklórica colombiana en niños de seis $y$ siete años en el contexto escolar. [Tesis doctoral]. Santiago de Cali, Colombia.

Cidoncha, V. y Díaz, E. (2009). Importancia del desarrollo del esquema corporal. Revista digital enfoques educativos, 13. (128).

Duran, S. y Parra, M. (2014). Diversidad Cultural para promover el desarrollo de habilidades sociales en educación superior. Cultura, Educación y Sociedad, 5(1), 55-67.

Escobar, C. (s.f). Danzas Lúdicas para Preescolar. A ritmo de nuestro folclor. Bogotá, D.C.: Editorial Magisterio.

Florián, M. (2015). Expresión corporal. El cuerpo como elemento fundamental de la expresión. Vigo: Ideaspropias.

Jiménez, C. (2000). Hacia la construcción del concepto de lúdica. Bogotá, D.C.: Editorial Magisterio.

Grawitz, M. (2011). Métodos y técnicas de las ciencias sociales. México, D.F.: Fontana y Frey. Obtenido de http:// trabajosocialmazatlan.com/multimedia/files/InvestigacionPosgrado/Entrevista.pdf

Hernández Sampieri, C. F. (2006). Metodología de la investigación (4 ed.). México, D.F.: McGraw-Hill. Obtenido de https://investigar1.files.wordpress. com/2010/05/1033525612-mtis_sampieri_unidad_1- 
Herrera, B., Certain, R. y Calderón, M (2014). La norma hipotética fundamental desde el Paradigma Interpretativo. Justicia Juris, 10(2), 17-27.

Jiménez, C. (2005). La inteligencia lúdica. Bogotá, D.C.: Editorial Magisterio.

Jutinico, F., Olaya C., Sotelo, J. y Vargas, M. (2011). Desarrollo de la expresión y la creatividad por medio de la gráfica en niños de preescolar. Universidad Minuto de Dios, Bogotá, D.C.

Martínez, E. (2014). Desarrollo psicomotor en educación infantil. Bases para la intervención en psicomotricidad. [Tesis]. Universidad Almería, Almería.

Martínez, M. (2006). La Investigación Cualitativa (Síntesis Conceptual). Revista IIPSI 9(1). 123 - 146. Recuperado de http://sisbib.unmsm.edu.pe/bvrevistas/investigacion_psicologia/v09_n1/ pdf/a09v9n1.pdf

Molina, M. (2014). Proyecto taller de dan$z a$. Recuperado de http://www.nuevaespana.cl/wp-content/uploads/2014/08/ Proyecto-Taller-de-Danza-2014.-pdf.pdf

Núñez Flórez, E., Vergara Ortiz, R., \& Bocanegra García, J. (2014). Sistema experto basado en lógica difusa tipo 1 para determinar el grado de riesgo de preeclampsia. INGE CUC, 10(1), 43-50. Recuperado de https://revistascientificas. cuc.edu.co/ingecuc/article/view/341
Posada, P. (2000). Cantar, tocar y jugar. Artes la revista, 1(1). Recuperado de http://aprendeenlinea.udea.edu.co/revistas/index.php/artesudea/article/ view/22095/18316

República de Colombia. Congreso de la República. (7 de agosto de 1997). Por la cual se desarrollan los artículos 70, 71 y 72 y demás artículos concordantes de la Constitución Politica y se dictan normas sobre patrimonio cultural, fomentos y estímulos a la cultura, se crea el Ministerio de la Cultura y se trasladan algunas dependencias. [Ley 397]. DO: 43102.

Romero-Acosta, K. y Contreras Banques, E. (2015). Revisión teórica sobre el postconflicto: una oportunidad para empoderar a mujeres víctimas de desplazamiento. Cultura Educación y Sociedad, 6(1). 79-92. Recuperado de https://revistascientificas.cuc.edu.co/ culturaeducacionysociedad/article/ view/757

Romero, G., Suárez, R. y Rodríguez, H. (2018). Modelo de capacidades de innovación para instituciones de educación superior. INGE CUC, 14(1), 87-100. https://doi.org/10.17981/ingecuc.14.1.2018.8

VAHOS, O. Juguemos, Cultura para la Paz: Medellín: Lito Roca. 